OPEN ACCESS

Edited by:

Brian Godman,

Karolinska Institutet (KI), Sweden

Reviewed by:

Amanj Kurdi,

University of Strathclyde,

United Kingdom

Johanna Catharina Meyer,

Sefako Makgatho Health Sciences

University, South Africa

*Correspondence:

Danielle M. van der Laan

d.vanderlaan1@vumc.nl

Specialty section:

This article was submitted to

Pharmaceutical Medicine

and Outcomes Research,

a section of the journal

Frontiers in Pharmacology

Received: 10 July 2018 Accepted: 03 September 2018

Published: 26 September 2018

Citation:

van der Laan DM, Elders PJM, Boons CCLM, Nijpels G, van Dijk L

and Hugtenburg JG (2018)

Effectiveness of a Patient-Tailored,

Pharmacist-Led Intervention Program

to Enhance Adherence

to Antihypertensive Medication:

The CATI Study.

Front. Pharmacol. 9:1057.

doi: 10.3389/fphar.2018.01057

\section{Effectiveness of a Patient-Tailored, Pharmacist-Led Intervention Program to Enhance Adherence to Antihypertensive Medication: The CATI Study}

Danielle M. van der Laan ${ }^{1 *}$, Petra J. M. Elders², Christel C. L. M. Boons ${ }^{1}$, Giel Nijpels², Liset van Dijk ${ }^{3}$ and Jacqueline G. Hugtenburg ${ }^{1}$

${ }^{1}$ Department of Clinical Pharmacology and Pharmacy and the Amsterdam Public Health Research Institute, VU University Medical Center, Amsterdam, Netherlands, ${ }^{2}$ Department of General Practice and Elderly Care Medicine and the Amsterdam Public Health Research Institute, VU University Medical Center, Amsterdam, Netherlands, ${ }^{3}$ Netherlands Institute for Health Services Research, Utrecht, Netherlands

Introduction: Non-adherence to medication is a complex health care problem. In spite of substantial efforts, up till now little progress has been made to effectively tackle the problem with adherence-enhancing interventions. The aim of this study was to investigate the effectiveness of a patient-tailored, pharmacist-led and theory-driven intervention program aimed to enhance self-reported adherence to antihypertensive medication.

Materials and Methods: A parallel-group randomized controlled trial in 20 community pharmacies with nine months follow-up was conducted. Patients (45-75 years) using antihypertensive medication and considered non-adherent based on both pharmacy dispensing data and a self-report questionnaire were eligible to participate. The intervention program consisted of two consultations with the pharmacist to identify participants' barriers to adhere to medication and to counsel participants in overcoming these barriers. The primary outcome was self-reported medication adherence. Secondary outcomes were beliefs about medicines, illness perceptions, quality of life and blood pressure. Mixed-model and generalized estimating equation (GEE) analyses were used to assess overall effects of the intervention program and effects per time point.

Results: 170 patients were included. No significant differences between intervention and control groups were found in self-reported adherence, quality of life, illness perceptions, beliefs about medicines (concern scale), and blood pressure. After nine months, intervention participants had significantly stronger beliefs about the necessity of using their medicines as compared to control participants (mean difference 1.25 [95\% Cl: 0.27 to 2.24], $p=0.012$ ). 
Discussion: We do not recommend to implement the intervention program in the current form for this study population. Future studies should focus on how to select eligible patient groups with appropriate measures in order to effectively target adherence-enhancing interventions.

Trial Register: NTR5017 http://www.trialregister.nl/trialreg/admin/rctview.asp?TC= 5017.

Keywords: medication adherence, patient-tailored, community pharmacies, randomized controlled trial, antihypertensive medication

\section{INTRODUCTION}

Non-adherence to medication is an ever continuing complex and multidimensional health care problem. Adherence is defined as the process by which patients take their medication as agreed upon with their health care provider, composed of initiation, implementation and discontinuation (Vrijens et al., 2012). At these three phases non-adherent behavior could occur. Patients may not take the first dose of a prescribed medicine, deviate from the prescribed dosing regimen for example by taking less or skipping pills or discontinue treatment prematurely. Research shows that adherence to antihypertensive medication is suboptimal (Kronish et al., 2011; Naderi et al., 2012; Abegaz et al., 2017). This results in poorly controlled hypertension, higher risk of cardiovascular events, and increased health care costs (Dragomir et al., 2010; Chowdhury et al., 2013; Kim et al., 2016). In Netherlands, community pharmacists are in the ideal position to address adherence, since they are medicines experts in primary care and the majority of patients receive their medication from a community pharmacy. In the past decades, numerous interventions in community pharmacies have been developed in order to increase medication adherence and improve treatment results (Altowaijri et al., 2013; Cheema et al., 2014). Unfortunately, in most studies the results on the effectiveness of these (pharmacist-led) interventions were disappointing (McDonald et al., 2002; Kripalani et al., 2007; van Dulmen et al., 2007; Linn et al., 2011; Vervloet et al., 2012; Nieuwlaat et al., 2014). In only half of the studies the medication adherence was increased and in only a few studies the intervention led to improved treatment outcomes. One likely explanation is that most studies did not use a theoretical framework, crucial for understanding the complexities of adherence behavior. On top of that, most described interventions did not made an effort to apply a patient-tailored approach for identifying the specific causes or barriers for individual patients. Thus, in spite of substantial efforts, up till now little progress has been made to effectively tackle the persistent problem of medication nonadherence.

Because non-adherence is caused by multiple factors, categorized by the World Health Organization (WHO) as

Abbreviations: CATI, cardiovascular medication non-adherence tailored intervention; EMERGE, ESPACOMP medication adherence reporting guidelines; QBS, quick barrier scan; SFK, foundation for pharmaceutical statistics; TIG, tailored intervention guide. social/economic, patient-, condition-, therapy-, health care team, and system-related factors (Sabate, 2003), interventions should be patient-tailored and designed according to a theoretical framework. A proven effective framework for behavioral change is the Common Sense Model of Self-regulation (Leventhal et al., 1984, 1992, 1997; Diefenbach and Leventhal, 1996). According to this theory it is thought that patients seek to understand their illness by developing a representation of illness and treatment, which guides their health behavior. For example, if a patient regards hypertension as a problem, the patient will adopt healthrelated behavior (such as taking antihypertensive medication) in order to cope with the problem. Thus, adherence behavior will be influenced by whether it makes sense given patients' illness and treatment representations (Meyer et al., 1985; Leventhal et al., 1992; Horne and Weinman, 1998, 2002; Reynolds, 2003; Rajpura and Nayak, 2014). Moreover, given the asymptomatic nature of hypertension and the need for long-term medicine use, patients' understanding of the illness may be important in achieving long-term medication adherence (Meyer et al., 1985).

Cardiovascular medication non-Adherence Tailored Intervention is a patient-tailored and pharmacist-led intervention program aimed to enhance self-reported adherence to antihypertensive medication, based on the Common Sense Model of Self-regulation (Van der Laan et al., 2017). The CATI intervention program includes informing participants about hypertension and its consequences in order to change illness and treatment representations, identifying participants' barriers to adhere to medication and providing recommendations and deciding on interventions to overcome the identified barriers. In this paper, we report on the effectiveness of the CATI intervention program to enhance self-reported medication adherence compared to usual care.

\section{MATERIALS AND METHODS}

\section{Study Design and Setting}

We performed a pragmatic parallel-group randomized controlled trial in 20 community pharmacies in rural and urban regions in Netherlands. Since this trial was pragmatic including more pharmacies was not possible. We included patients between March and June 2016 and followed them for nine months. Patients were randomly assigned to the intervention group executed by the pharmacist or control group executed by the pharmacy technician (allocation ratio 1:1). Blinding to treatment 
allocation was not possible due to the nature of the intervention. The study protocol has been described in more detail elsewhere (Van der Laan et al., 2017). The Medical Ethics Committee of the VU University Medical Center approved the study, and all participants gave written informed consent. The study was performed in accordance with the Declaration of Helsinki (2008) and the Dutch Medical Research involving Human Subjects Act (WMO). The trial was registered in the Dutch Trial Register (NTR5017). For this paper, the EMERGE were followed (De Geest et al., 2018).

\section{Study Population}

Patients aged 45-75 years using antihypertensive medication (including beta-blockers, calcium antagonists, diuretics, angiotensin converting enzyme (ACE) inhibitors, and angiotensin II-receptor antagonists) for at least twelve months and indicating to have hypertension by self-report were eligible to participate. We excluded patients who had insufficient Dutch language skills or used medication-intake supporting services provided by the pharmacy, i.e., repeat dispensing and pill packaging.

Patients non-adherent according to pharmacy dispensing data were selected using the selection method of the SFK, which is developed by the Royal Dutch Pharmacists Association (SFK, 2013). SFK registers information on dispensed drugs and calculates the Proportion of Days Covered (PDC). Patients with a PDC $<80 \%$ for one antihypertensive drug class during the last six months were considered non-adherent (Nau, 2012). In each pharmacy a random sample of 75 patients was selected for inclusion using a randomization table. The selected patients were invited to participate and received the baseline questionnaire including the Medication Adherence Report Scale (MARS-5) (Horne, 2013). The MARS-5 assessed patients self-reported nonadherence to antihypertensive medication. Patients willing to participate were included if they were non-adherent based on both pharmacy dispensing data (PDC $<80 \%)$ and the self-report

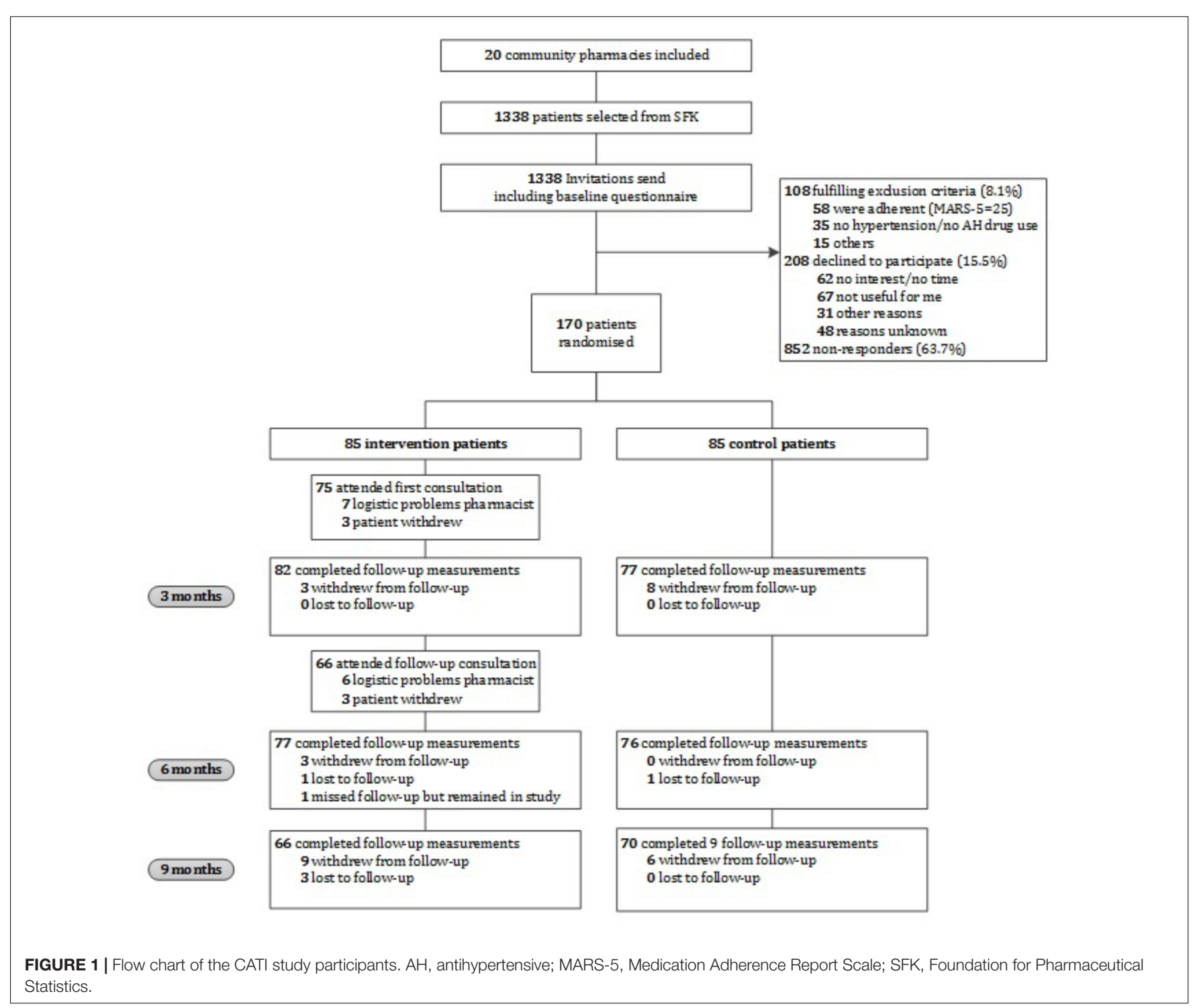


questionnaire (MARS-5 <25). This study focused on medication adherence in the implementation phase of treatment (Vrijens et al., 2012).

\section{Intervention Group}

Participants in the intervention group received the CATI intervention program in addition to usual care. The program was executed by the pharmacist and included two consultations in the pharmacy. Participating pharmacists received a training on how to deliver the intervention program.

\section{First Consultation}

The first consultation started with a semi-structured interview, called the QBS (Van der Laan et al., 2017). The aim of the QBS was to explore participants' barriers to adhere to medication assessed with twelve questions. Based upon the identified barrier(s) at least one corresponding intervention module was selected according to the TIG (Van der Laan et al., 2017). The TIG contains an overview of intervention recommendations divided in five intervention modules: (1) Providing Information, (2) Providing Tools, (3) Dealing with Side Effects, (4) Overcoming Practical Problems, and (5) Diminishing Negative Beliefs. The first consultation continued with discussing participants' illness and treatment representations and providing tailored information regarding potential risks of hypertension, use of medication and living a healthy lifestyle. Herewith, participants' understanding of hypertension and the perceived need to be adherent to antihypertensive treatment would be increased, which is emphasized as important for achieving long-term medication adherence by the Common Sense Model of Self-regulation (Meyer et al., 1985). Subsequently, the pharmacist provided the participant with tailored recommendations from the selected intervention module to overcome the identified barriers. Finally, the participant received a written summary of the consultation including the information and recommendations provided.

\section{Follow-Up Consultation}

Approximately three months after the first consultation, a follow-up consultation was planned with the participant. The purpose of this follow-up consultation was to discuss participants' implementation of and experiences with the discussed information and recommendations.

\section{Control Group}

Participants in the control group only received usual care according to the Dutch guidelines of the Royal Dutch Pharmacists Association (KNMP, 2006). This care, usually delivered by a pharmacy technician, consist of checking and dispensing of prescribed drugs, providing instructions on medication use, and providing information about intended effects and possible side effects, during first and second dispensing. Blood pressure measurements in the control group were executed by the pharmacy technician.

\section{Outcome Measures}

Both intervention and control group participants received a questionnaire by post at baseline and after three, six and nine months. This comprehensive questionnaire assessed medication adherence, beliefs about medicines, quality of life and illness perceptions.

The primary outcome was self-reported medication adherence assessed with MARS-5 (Horne, 2013). The MARS-5 comprises five statements of adherence-related behavior: "I alter the dose of my medicines," "I stop taking my medicines for a while," "I decide to miss out on a dose of my medicines," "I forget to take my medicines," and "I take less of my medicines than instructed." Each statement is rated on a five-point scale, from 1 (always) to 5 (never). The MARS-5 sum score was calculated, ranging from 5 to 25 points where a higher score indicates better adherence. The MARS- 5 sum score was also dichotomized, where a score below 25 points indicates non-adherence. The MARS5 questionnaire is an easy-to-use tool and shows acceptable reliability and validity (Horne et al., 2001; Cohen et al., 2009; Mora et al., 2011; Bäck et al., 2012; Salt et al., 2012; Horne, 2013; Lin et al., 2018).

Secondary outcomes included participants' beliefs about medicines assessed with the validated and reliable Specific Beliefs about Medicines Questionnaire (BMQ) (Horne and Weinman, 1999a; Horne et al., 1999b). The BMQ consists of ten questions scored on a 5-point Likert scale and is subdivided into a Necessity scale and Concern scale, both ranging from 5 to 25 points.

Participants' quality of life was assessed with the validated and reliable 12-Item Short Form (SF-12) questionnaire (Hurst et al., 1998). The SF-12 questionnaire consists of twelve questions covering eight domains of health. The eight domains produces two summary scores including physical health (PCS) and mental health (MCS) both ranging from 0 to 100.

Participants' illness perceptions were assessed using the reliable and validated Brief Illness Perceptions Questionnaire (Brief IPQ-9) (Broadbent et al., 2006). The IPQ-9 is a nine-item scale designed to assess participants' cognitive and emotional representations of illness. All items except the causal question are rated on a 0 to 10 point scale.

Participants' systolic and diastolic blood pressure was measured three times during the nine months follow-up according to a standardized protocol. In the intervention group the pharmacist performed the measurements at the first consultation, at the follow-up consultation and at a final pharmacy visit. For the control group the blood pressure was measured by a pharmacy technician during three additionally scheduled pharmacy visits. At each visit the blood pressure was measured three times, each two minutes apart, in seated position with an automatic sphygmomanometer (Lee et al., 2006). Measured blood pressure was calculated as the mean of the second and third measurement values.

\section{Sample Size}

A sample size calculation for proportions, as described in the study protocol (Van der Laan et al., 2017), was performed on a clinical relevant difference of $20 \%$ in percentage of adherent participants on the self-reported MARS-5. Based on this calculation a total sample size of 156 participants was required. 
TABLE 1 | Baseline characteristics of participants of the CATI study.

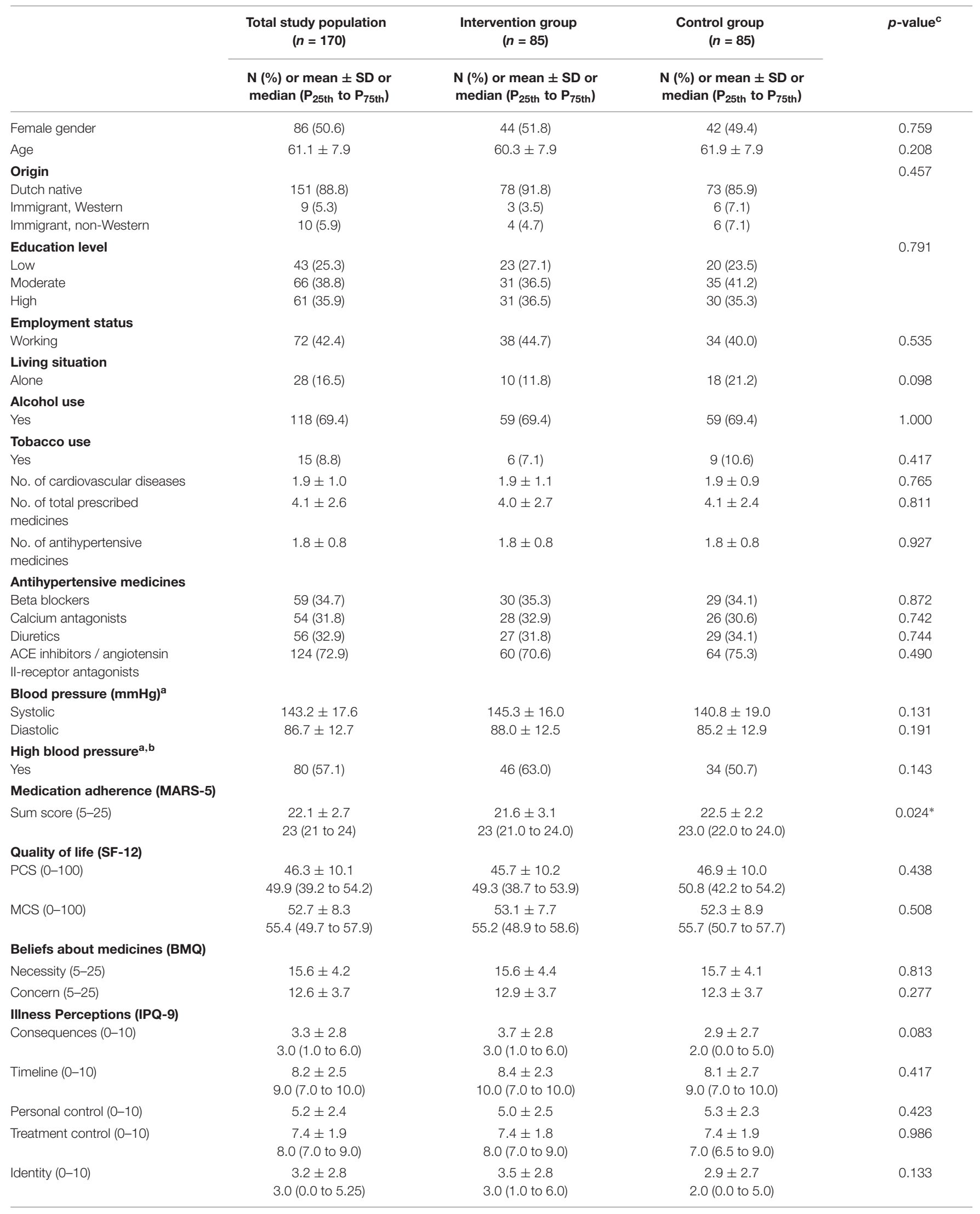


TABLE 1 | Continued

\begin{tabular}{|c|c|c|c|c|}
\hline & $\begin{array}{l}\text { Total study population } \\
\qquad(n=170)\end{array}$ & $\begin{array}{l}\text { Intervention group } \\
\qquad(n=85)\end{array}$ & $\begin{array}{l}\text { Control group } \\
\qquad(n=85)\end{array}$ & $p$-value ${ }^{c}$ \\
\hline & $\begin{array}{l}N(\%) \text { or mean } \pm S D \text { or } \\
\text { median }\left(P_{25 \text { th }} \text { to } P_{75 t h}\right)\end{array}$ & $\begin{array}{l}N(\%) \text { or mean } \pm S D \text { or } \\
\text { median }\left(P_{25 \text { th }} \text { to } P_{75 t h}\right)\end{array}$ & $\begin{array}{l}N(\%) \text { or mean } \pm S D \text { or } \\
\text { median }\left(P_{25 \text { th }} \text { to } P_{75 t h}\right)\end{array}$ & \\
\hline |llness concerns (0-10) & $4.7 \pm 2.8$ & $4.7 \pm 2.9$ & $4.7 \pm 2.8$ & 0.978 \\
\hline Coherence (0-10) & $6.0 \pm 2.7$ & $5.9 \pm 3.0$ & $6.1 \pm 2.4$ & 0.548 \\
\hline Emotional representation (0-10) & $\begin{array}{c}2.7 \pm 2.7 \\
2.0(0.0 \text { to } 5.0)\end{array}$ & $\begin{array}{c}2.7 \pm 2.5 \\
2.0(0.0 \text { to } 5.0)\end{array}$ & $\begin{array}{c}2.6 \pm 2.9 \\
2.0(0.0 \text { to } 4.0)\end{array}$ & 0.842 \\
\hline
\end{tabular}

TABLE 2A | Mean scores \pm SD and proportions (\%) of the primary and secondary outcomes at each measurement point.

\begin{tabular}{|c|c|c|c|c|c|c|c|c|c|}
\hline & & \multicolumn{2}{|c|}{ Baseline } & \multicolumn{2}{|l|}{ T1 } & \multicolumn{2}{|l|}{ T2 } & \multicolumn{2}{|l|}{ T3 } \\
\hline & & Mean \pm SD & $n$ & Mean \pm SD & $n$ & Mean \pm SD & $n$ & Mean \pm SD & $n$ \\
\hline \multirow[t]{2}{*}{ MARS-5 sum score } & Intervention & $21.6 \pm 3.1$ & 85 & $22.5 \pm 2.5$ & 82 & $22.7 \pm 2.4$ & 77 & $22.8 \pm 2.3$ & 66 \\
\hline & Control & $22.5 \pm 2.2$ & 85 & $23.0 \pm 1.9$ & 77 & $22.9 \pm 2.2$ & 76 & $23.1 \pm 2.3$ & 70 \\
\hline & Control & $85(100)$ & 85 & $64(83.1)$ & 77 & 65 (85.5) & 76 & 55 (78.6) & 70 \\
\hline & & Mean \pm SD & $n$ & Mean \pm SD & $n$ & Mean \pm SD & $n$ & Mean \pm SD & $n$ \\
\hline \multicolumn{10}{|l|}{ Secondary outcomes } \\
\hline SF-12 PCS (0-100) & Intervention & $45.7 \pm 10.2$ & 85 & $45.5 \pm 9.6$ & 82 & $46.0 \pm 10.3$ & 77 & $46.0 \pm 10.2$ & 66 \\
\hline \multirow[t]{2}{*}{ BMQ Necessity (5-25) } & Intervention & $15.6 \pm 4.4$ & 85 & $15.8 \pm 4.3$ & 82 & $16.2 \pm 4.5$ & 77 & $16.9 \pm 4.4$ & 66 \\
\hline & Control & $15.7 \pm 4.1$ & 85 & $15.7 \pm 4.0$ & 77 & $16.1 \pm 4.5$ & 76 & $16.0 \pm 4.2$ & 70 \\
\hline \multirow[t]{2}{*}{ BMC Concern (5-25) } & Intervention & $12.9 \pm 3.7$ & 85 & $13.1 \pm 3.9$ & 82 & $12.5 \pm 4.1$ & 77 & $12.6 \pm 3.9$ & 66 \\
\hline & Control & $12.3 \pm 3.7$ & 85 & $12.7 \pm 3.9$ & 77 & $12.3 \pm 4.4$ & 76 & $12.4 \pm 3.9$ & 70 \\
\hline \multirow[t]{2}{*}{ IPQ-9 Consequences (0-10) } & Intervention & $3.7 \pm 2.8$ & 85 & $3.3 \pm 2.5$ & 82 & $3.5 \pm 2.7$ & 77 & $3.7 \pm 2.8$ & 66 \\
\hline & Control & $2.9 \pm 2.7$ & 85 & $3.3 \pm 2.6$ & 77 & $3.5 \pm 2.7$ & 76 & $3.4 \pm 2.8$ & 70 \\
\hline \multirow[t]{2}{*}{ IPQ-9 Timeline (0-10) } & Intervention & $8.4 \pm 2.3$ & 84 & $8.5 \pm 2.0$ & 81 & $8.4 \pm 2.3$ & 77 & $8.5 \pm 2.4$ & 66 \\
\hline & Control & $8.1 \pm 2.7$ & 85 & $8.1 \pm 2.5$ & 75 & $7.9 \pm 2.6$ & 76 & $8.2 \pm 2.4$ & 69 \\
\hline \multirow[t]{2}{*}{ IPQ-9 Personal control (0-10) } & Intervention & $5.0 \pm 2.5$ & 85 & $5.6 \pm 2.3$ & 81 & $5.4 \pm 2.4$ & 77 & $5.6 \pm 2.3$ & 66 \\
\hline & Control & $5.3 \pm 2.3$ & 85 & $5.8 \pm 2.3$ & 76 & $5.8 \pm 2.4$ & 76 & $5.9 \pm 2.3$ & 69 \\
\hline IPQ-9 Treatment control (0-10) & Intervention & $7.4 \pm 1.8$ & 84 & $7.5 \pm 2.0$ & 81 & $7.4 \pm 2.2$ & 77 & $7.6 \pm 1.8$ & 66 \\
\hline \multirow[t]{2}{*}{ IPQ-9 Emotional representation (0-10) } & Intervention & $2.7 \pm 2.5$ & 85 & $2.6 \pm 2.4$ & 81 & $2.7 \pm 2.7$ & 77 & $3.3 \pm 2.9$ & 66 \\
\hline & Control & $2.6 \pm 2.9$ & 85 & $2.6 \pm 2.5$ & 76 & $2.9 \pm 2.9$ & 76 & $2.9 \pm 2.8$ & 70 \\
\hline
\end{tabular}

BMQ, Specific Beliefs about Medicines Questionnaire; IPQ-9, IIIness Perceptions Questionnaire; MARS-5, Medication Adherence Report Scale; MCS, Mental Component Score; PCS, Physical Component Score; SD, standard deviation, SF-12, 12-item Short Form. 
TABLE 2B | Mean scores \pm SD of systolic and diastolic blood pressure measurements at three visits during study period.

\begin{tabular}{|c|c|c|c|c|c|c|c|}
\hline & & \multicolumn{2}{|c|}{ Visit 1 (baseline) } & \multicolumn{2}{|c|}{ Visit 2} & \multicolumn{2}{|c|}{ Visit 3} \\
\hline & & Mean \pm SD & $n$ & Mean \pm SD & $n$ & Mean \pm SD & $n$ \\
\hline \multicolumn{8}{|l|}{ Secondary outcomes } \\
\hline \multirow[t]{2}{*}{ Systolic blood pressure } & Intervention & $145.3 \pm 16.0$ & 73 & $142.9 \pm 19.0$ & 66 & $145.1 \pm 16.7$ & 55 \\
\hline & Control & $140.8 \pm 19.0$ & 67 & $140.0 \pm 17.8$ & 65 & $142.9 \pm 17.4$ & 55 \\
\hline \multirow[t]{2}{*}{ Diastolic blood pressure } & Intervention & $88.0 \pm 12.5$ & 73 & $88.1 \pm 13.9$ & 66 & $87.3 \pm 13.6$ & 55 \\
\hline & Control & $85.2 \pm 12.9$ & 67 & $85.6 \pm 12.5$ & 65 & $86.7 \pm 12.2$ & 55 \\
\hline
\end{tabular}

SD, standard deviation.

\section{Statistical Analyses}

Descriptive statistics were used to describe patient characteristics. Means and standard deviations for continuous variables and frequencies and percentages for categorical variables were presented. For non-normal continuous outcomes medians with interquartile ranges were also provided. To compare baseline values between the intervention and control group independent sample $t$-tests and chi-square tests were used. The effect analyses of the intervention program by comparing the differences between the intervention and control group were performed according to the "intention-to-treat" principle. Linear mixedmodel analyses were used for continuous outcomes, and logistic generalized estimating equation (GEE) analyses were used for dichotomous outcomes. Logistic GEE analyses were preferred over logistic mixed-model analyses because of the instability of the latter and the overestimation of the effect estimates (Twisk, 2006). For all outcomes both an overall effect of the intervention program and effects of the intervention program at the different time points were estimated (three, six, and nine months). For each outcome variable crude regression coefficients were calculated (only adjusted for the baseline value of the particular outcome), as well as adjusted regression coefficients (adjusted for the baseline value of the particular outcome, the MARS-5 baseline score and possible confounders age, gender and education level). For the GEE analyses both crude and adjusted odds ratios (OR) were calculated. In addition to the main analyses, several sensitivity analyses were performed. First, a "per protocol" analyses was performed including all intervention participants that did visit the pharmacy for both the first and follow-up consultation. Second, several subgroup analyses were performed to stratify for gender, younger (45-55) and older (56-75) age and normal and high blood pressure measured at the first visit (baseline). Another subgroup analysis was performed including the intervention participants with $\geq 3$ barriers identified with the QBS during the first consultation. A final subgroup analysis was performed using a more rigorous cut-off value for the primary outcome, including participants with a MARS-5 score of $\leq 23$ at baseline (Mardby et al., 2007; Sjolander et al., 2013). A score of $>23$ was considered adherent. Mixed-model and GEE analyses were performed using Stata/SE version 14.0 (StataCorp LP, College Station, TX, United States). All other analyses were performed using SPSS version 22.0 (IBM Corp, Armonk, NY, United States).

\section{RESULTS}

\section{Baseline Characteristics}

Figure 1 provides an overview of the flow of participants through the study. In total, 170 participants were included ( 85 in the intervention group and 85 in the control group). At baseline, the MARS-5 score was 0.9 points lower in the intervention group as compared to the control group, which indicates that the intervention group was significantly less adherent than the control group (mean difference -0.9 [95\% CI: -1.76 to -0.12 ], $p=0.024$ ) (Table 1). No other relevant differences with respect to participant characteristics between the intervention and control group were found (Table $\mathbf{1}$ ).

\section{Declined to Participate and Non-responders}

In total, 1338 patients were invited to participate. Of them, 108 (8.1\%) did not meet the inclusion criteria, 208 (15.5\%) declined to participate, and $852(63.7 \%)$ did not respond (Figure 1). Patients who declined to participate did not differ in gender from participants, however, they were significantly older $(p \leq 0.001)$ and had a lower level of education $(p=0.005)$. Reasons indicated for non-participation were no interest, no time, or not needed according to the patient. Gender and age of non-responders were comparable to participants.

\section{Intervention Program}

The first consultation of the intervention program was held with 75 participants $(88.2 \%)$ and the follow-up consultation with 66 participants (77.6\%). The average period between the first and follow-up consultation was 94 days. In most cases missed consultations were due to logistic and time management problems of pharmacists. The average time of the first and follow-up consultation was 36 min (range: 15$85 \mathrm{~min}$ ) and $20 \mathrm{~min}$ (range: $5-45 \mathrm{~min}$ ), respectively. Participants who did not attend both consultations did not differ in age, gender, origin, education level, employment status or living situation from participants who did attend both consultations, however, they used significantly more antihypertensive medicines $(p=0.044)$.

\section{Primary Outcome}

Table 2A presents the mean scores and proportions of the primary outcome. In the "intention-to-treat" analysis, no 


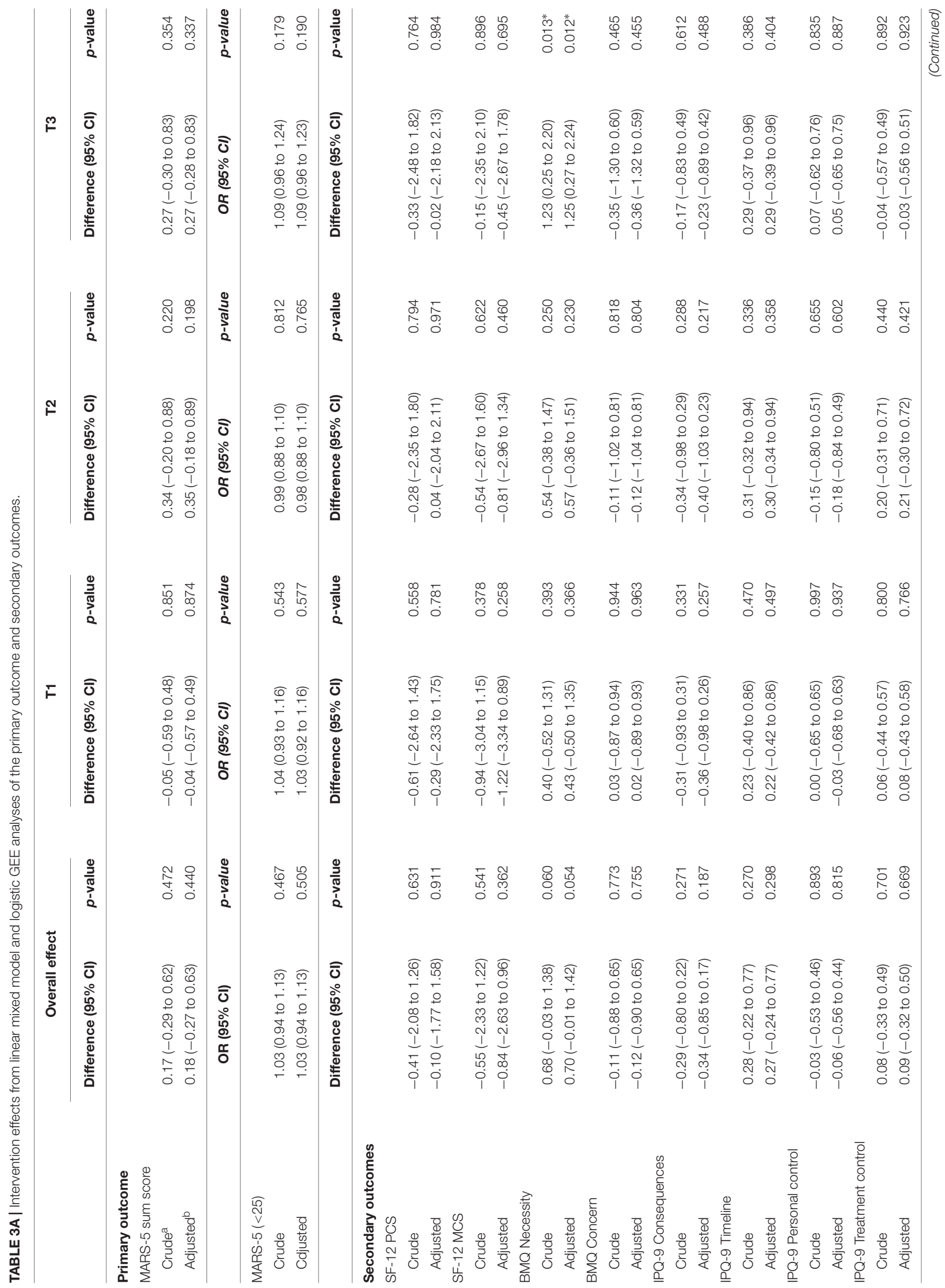



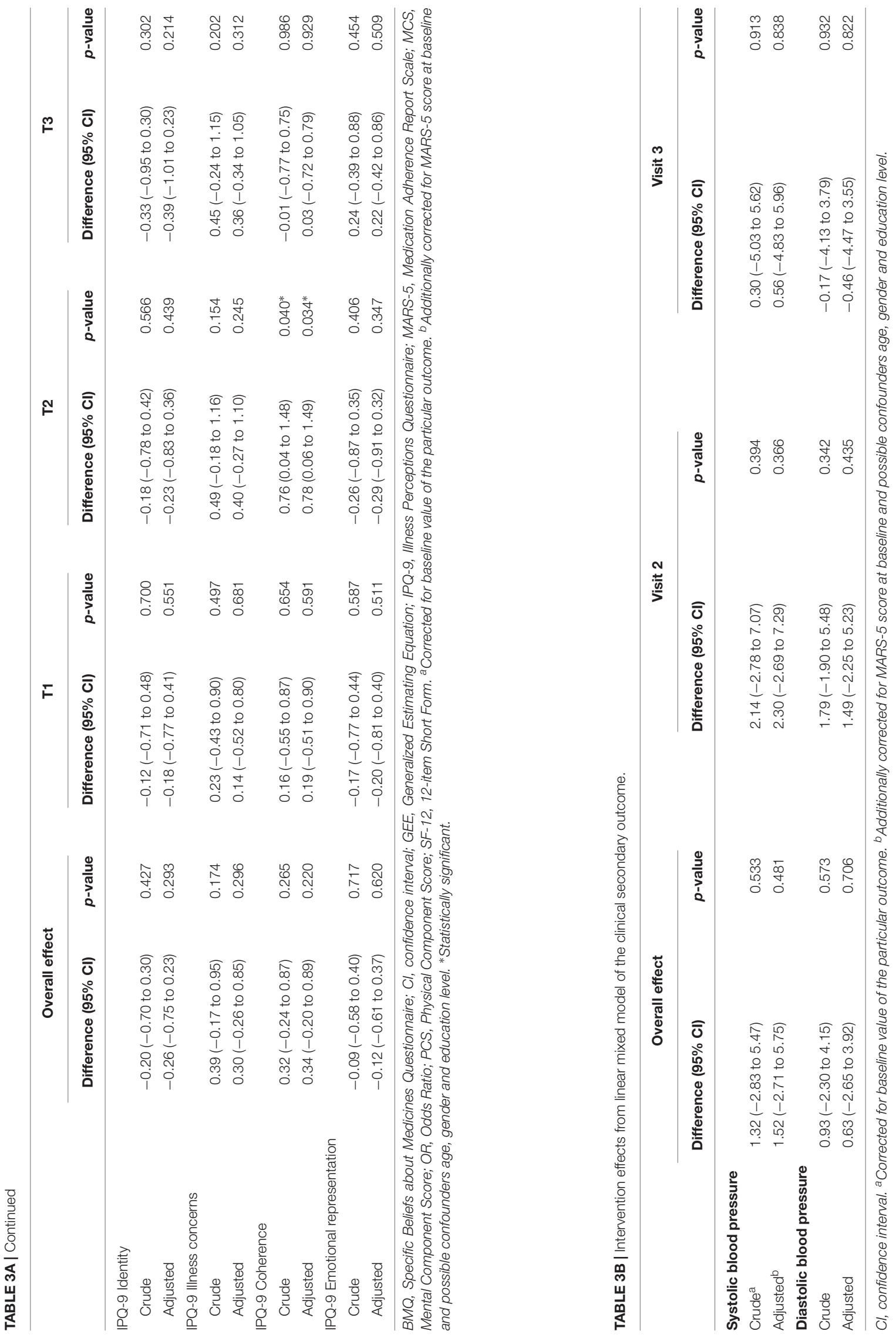
significant differences were found in self-reported adherence over time or after three, six and nine months between the intervention and control group (Table 3A). Results of the sensitivity analyses are presented in Appendix A. Per protocol and most subgroup analyses did not show different results. However, in the subgroup analysis in which we only included participants with $\geq 3$ barriers identified during the first consultation, a significant intervention effect was found on self-reported medication adherence after nine months (mean difference 0.84 [95\% CI: 0.03 to 1.65], $p=0.042$ ). In the subgroup analysis where we used a more rigorous cut-off value for the MARS-5 score, a more positive intervention effect was found (mean difference 0.43 [95\% CI: -0.30 to 1.15$]$ ), however, not statistically significant.

\section{Secondary Outcomes}

In Tables 2A,B the mean scores of the secondary outcomes are listed. For the quality of life (SF-12), the illness perceptions (IPQ9) and the systolic and diastolic blood pressure, no significant differences over time or at the different time points between the intervention and control group were found (Tables $\mathbf{3 A}, \mathbf{B}$ ). For the beliefs about medicines, a borderline significant difference over time was found on the BMQ Necessity scale (Tables 3A,B). This overall effect was found because participants in the intervention group had a significantly higher BMQ Necessity score at nine months follow-up (mean difference 1.25 [95\% CI: 0.27 to 2.24 ], $p=0.012$ ). This means that they had significantly stronger beliefs about the necessity of using their medicines as compared to the control group.

\section{DISCUSSION}

The aim of this study was to evaluate the effectiveness of the CATI intervention program to enhance self-reported adherence to antihypertensive medication as compared to usual care. There were no statistically significant effects in adherencerelated behavior, quality of life, illness perceptions, beliefs about medicines (concern scale), and blood pressure. For the beliefs about medicines, we did find a statistical significant intervention effect on the BMQ Necessity scale after nine months.

A systematic review of Gwadry-Sridhar et al. (2013) reported that only $35.1 \%$ of comparable intervention studies aimed to directly improve adherence in patients with hypertension did significantly improve medication adherence. In studies with quite comparable intervention programs inconsistent findings were reported (Morgado et al., 2011; Stewart et al., 2014; Nguyen et al., 2016). For example, in a study of Morgado et al. (2011) a pharmaceutical care program including two counseling visits to the pharmacy in patients using antihypertensive medication was evaluated. They reported statistically significant effects on both blood pressure control and self-reported medication adherence at nine months. In a study of Stewart et al. (2014) patients visited the pharmacy three times and received a package of interventions, including motivational interviewing, medication review and prescription refill reminders. They concluded that the intervention had a significant effect on blood pressure, but not on medication adherence. In a study of Nguyen et al. (2016) a multifaceted medication management intervention for patients with hypertension, type 2 diabetes or other cardiovascular disease significantly improved medication adherence. This study also found that intervention patients had significantly stronger necessity beliefs compared to control patients. This latter result is in line with the finding of our study.

There are several possible explanations for the absence of effects on medication adherence-related behavior in our study. First, the design of our study was a parallel-group randomized controlled trial in which individuals within one pharmacy were randomized into the intervention or control group. It might have been better to apply cluster randomization to control for contamination across groups. However, to avoid contamination in our study the intervention group was executed by the pharmacist, whereas the control group was executed by the pharmacy technician. Second, since large numbers of patients did not respond $(63.7 \%)$ and declined to participate $(15.5 \%)$ we cannot rule out selection bias. It might be possible that we missed patients that were unaware of their incompetence to properly use their medicines and therefore did not score non-adherent on the self-reported questionnaire. In addition, patients with strong negative beliefs toward medicines are probably not responsive for participation in intervention studies, while these patients are the population most interesting to target. With respect to drop-out, participants who did not attend the consultations used significantly more antihypertensive medicines compared to participants who did attend both consultations. This can be seen as a limitation, since patients with multiple medicines will benefit most from counseling interventions. However, we consider the bias of study results limited because in most cases the reason for not performing a consultation were logistic and time management problems of pharmacists. Third, the absence of effects could be related to the eligibility criteria of the study population, especially with regard to the outcome measure. There has been some debate about the appropriateness of MARS-5 as a medication adherence measure in the literature (van de Steeg et al., 2009; Tommelein et al., 2014). An explanation for these inconsistent results could be the use of pharmacy refill data as reference standard, since this measures another underlying construct compared to self-reported questionnaires. However, a large number of studies have shown acceptable validity and reliability of MARS-5 and therefore we have chosen this measure for this study (Cohen et al., 2009; Mora et al., 2011; Bäck et al., 2012; Salt et al., 2012; Horne, 2013; Lin et al., 2018). The chosen cut-off value for MARS-5 might be a plausible explanation for the absence of effects. A self-reported MARS5 score of below 25 points, indicates marginally non-adherent behavior. This resulted in too little room for improvement. Results of the sensitivity analysis on participants with lower self-reported adherence at baseline (MARS-5 $\leq 23$ ) were more positive than the main analysis. It may, therefore, be more efficient to target an intervention like this on patient groups with a lower degree of adherence. Another outcome measure limitation included that we were not able to collect pharmacy refill data after baseline. Because if a participant started repeat 
dispensing (medication-intake supporting service of pharmacy) during the study period, the dispensing was regulated and therefore participants could no longer be identified as nonadherent with the SFK selection method. Since pharmacy refill data could not be collected, we were not able to combine two different methods to assess medication adherence, a strategy that has been suggested in the literature (Sabate, 2003; Lam and Fresco, 2015). A patient group who experiences multiple barriers to adhere to medication and therefore might deal with more structural adherence problems, might also be more eligible for this kind of intervention. This is confirmed by the more positive and significant intervention effects in the sensitivity analyses on participants with $\geq 3$ identified barriers. Since only $57 \%$ of participants had an uncontrolled blood pressure at baseline, using uncontrolled blood pressure as an eligibility criterion in adherence-enhancing interventions must be considered. However, it might also be important to target both patients with or without uncontrolled blood pressure, since non-adherent behavior is continuous and dynamic and can occur over time (Sabate, 2003). Fourth, since we only found a significant effect in the main analyses on the BMQ Necessity scale after nine months, it might be possible that improvement of actual adherence-related behavior can only be expected after a longer period of time (Horne and Weinman, 1999a; Ross et al., 2004; Rajpura and Nayak, 2014). In the literature the Stages of Change model describes that healthrelated behavior change is rarely easy and requires a gradual progression of small steps (Prochaska and Velicer, 1997). In early stages, becoming more aware of the need for treatment, is an important factor for the beginning of small changes in behavior. It might, therefore, be more efficient to target patients in the initiation phase of medicine-taking (at the start of therapy). For instance, a study testing a pharmacist telephone counseling intervention found improvements in medication adherence in patients initiating therapy (Kooij et al., 2016). In contrast, it might also be efficient to target patients with long-term medication use, since these patients might experience treatment fatigue and therefore are at risk for medication non-adherence. Finally, since no effects were found on participants' illness perceptions, the use of the Common Sense Model of Self-regulation as a foundation of adherence-enhancing interventions must be reconsidered.

\section{Implications for Practice and Research}

Based on the results, we do not recommend to implement the CATI intervention program in the current form for the population selected in this study. The chosen cut-off value of MARS-5 might have been too liberal resulting in including

\section{REFERENCES}

Abegaz, T. M., Shehab, A., Gebreyohannes, E. A., Bhagavathula, A. S., and Elnour, A. A. (2017). Nonadherence to antihypertensive drugs: a systematic review and meta-analysis. Medicine 96:e5641. doi: 10.1097/MD.00000000000 05641

Altowaijri, A., Phillips, C. J., and Fitzsimmons, D. (2013). A systematic review of the clinica 1 and economic effectiveness of clinical pharmacist intervention participants with only marginally non-adherent behavior. Future studies should focus on how to select eligible patient groups with appropriate measures in order to effectively target adherence-enhancing interventions. Study populations with more severe non-adherent behavior and more barriers to adhere to medication, might benefit more from interventions, which is also confirmed by the more positive results of our sensitivity analyses. Future studies should also explore whether uncontrolled blood pressure should be added as an eligibility criterion. Moreover, future research might focus more on raising awareness and patients' necessity beliefs of using medicines, in order to change patients' adherence-related behavior. Finally, a process evaluation study would provide more insight into whether the ineffectiveness of the CATI intervention program was due to poor implementation or inadequacies of the intervention itself.

\section{AUTHOR CONTRIBUTIONS}

DvdL developed the study protocol, drafted the manuscript, coordinated data collection, analyzed the data, and reported the study results. $\mathrm{JH}$ and $\mathrm{CB}$ developed the study protocol, revised the manuscript, supported in data collection, analyzed the data, and reported the study results. $\mathrm{PE}, \mathrm{GN}$, and $\mathrm{LvD}$ participated in the design of the study and revised the manuscript. All authors read and approved the final manuscript.

\section{FUNDING}

This study was funded by the Royal Dutch Pharmacists Association (KNMP). The funding body did not have any role in trial design, collection, analysis, or interpretation of data, nor in writing the manuscript.

\section{ACKNOWLEDGMENTS}

We thank all patients, pharmacists, and pharmacy technicians who participated in the CATI study. We also thank Prof. J. W. R. Twisk for his support with the statistical data analyses.

\section{SUPPLEMENTARY MATERIAL}

The Supplementary Material for this article can be found online at: https://www.frontiersin.org/articles/10.3389/fphar. 2018.01057/full\#supplementary-material

in secondary prevention of cardiovascular disease. J. Manag. Care Pharm. 19, 408-416. doi: 10.18553/jmcp.2013.19.5.408

Bäck, A., Andersson Sundell, K., Horne, R., Landén, M., and Mårdby, A.-C. (2012). The medication adherence report scale (MARS-5) in a Swedish sample with bipolar disorder - a pilot study. Int. J. Pers. Cent. Med. 2, 263-270.

Broadbent, E., Petrie, K. J., Main, J., and Weinman, J. (2006). The brief illness perception questionnaire. J. Psychosom. Res. 60, 631-637. doi: 10.1016/j. jpsychores.2005.10.020 
Cheema, E., Sutcliffe, P., and Singer, D. R. (2014). The impact of interventions by pharmacists in community pharmacies on control of hypertension: a systematic review and meta-analysis of randomized controlled trials. Br. J. Clin. Pharmacol. 78, 1238-1247. doi: 10.1111/bcp.12452

Chowdhury, R., Khan, H., Heydon, E., Shroufi, A., Fahimi, S., Moore, C., et al. (2013). Adherence to cardiovascular therapy: a meta-analysis of prevalence and clinical consequences. Eur. Heart J. 34, 2940-2948. doi: 10.1093/eurheartj/ eht295

Cohen, J. L., Mann, D. M., Wisnivesky, J. P., Horne, R., Leventhal, H., MusumeciSzabo, T. J., et al. (2009). Assessing the validity of self-reported medication adherence among inner city asthmatic adults: the medication adherence report scale for asthma. Ann. Allergy Asthma Immunol. 103, 325-331. doi: 10.1016/ S1081-1206(10)60532-7

De Geest, S., Zullig, L. L., Dunbar-Jacob, J., Helmy, R., Hughes, D. A., Wilson, I. B., et al. (2018). ESPACOMP Medication Adherence Reporting Guideline (EMERGE). Ann. Intern. Med. 169, 30-35. doi: 10.7326/M18-0543

Diefenbach, M. A., and Leventhal, H. (1996). The common-sense model of illness representation: theoretical and practical considerations. J. Soc. Distress Homeless 5, 11-38. doi: 10.1007/BF02090456

Dragomir, A., Cote, R., Roy, L., Blais, L., Lalonde, L., Berard, A., et al. (2010). Impact of adherence to antihypertensive agents on clinical outcomes and hospitalization costs. Med. Care 48, 418-425. doi: 10.1097/MLR. 0b013e3181d567bd

Gwadry-Sridhar, F. H., Manias, E., Lal, L., Salas, M., Hughes, D. A., RatzkiLeewing, A., et al. (2013). Impact of interventions on medication adherence and blood pressure control in patients with essential hypertension: a systematic review by the ISPOR medication adherence and persistence special interest group. Value Health 16, 863-871. doi: 10.1016/j.jval.2013.03. 1631

Horne, R. (2013). The Medication Adherence Report Scale (MARS): a New Measurement Tool for Eliciting Patients' Report Non-adherence [Working paper]. Brighton: University of Brighton.

Horne, R., Hankins, M., and Jenkins, R. (2001). The Satisfaction with Information about Medicines Scale (SIMS): a new measurement tool for audit and research. Qual. Health Care 10, 135-140. doi: 10.1136/qhc.0100135

Horne, R., and Weinman, J. (1998). "Predicting treatment adherence: an overview of theoretical models," in Adherence to Treatment in Medical Conditions, eds L. B. Myers and K. Midence (Amsterdam: Harwood Academic Publishers).

Horne, R., and Weinman, J. (1999a). Patients' beliefs about prescribed medicines and their role in adherence to treatment in chronic physical illness. J. Psychosom. Res. 47, 555-567.

Horne, R., and Weinman, J. (2002). Self-regulation and self-management in asthma: exploring the role of illness perceptions and treatment beliefs in explaining non-adherence to preventer medication. Psychol. Health 17, 17-32. doi: 10.1080/08870440290001502

Horne, R., Weinman, J., and Hankins, M. (1999b). The beliefs about medicines questionnaire: the development and evaluation of a new method for assessing the cognitive representation of medication. Psychol. Health 14, 1-24. doi: 10. 1080/08870449908407311

Hurst, N. P., Ruta, D. A., and Kind, P. (1998). Comparison of the MOS short form12 (SF12) health status questionnaire with the SF36 in patients with rheumatoid arthritis. Br. J. Rheumatol. 37, 862-869. doi: 10.1093/rheumatology/37.8.862

Kim, S., Shin, D. W., Yun, J. M., Hwang, Y., Park, S. K., Ko, Y. J., et al. (2016). Medication adherence and the risk of cardiovascular mortality and hospitalization among patients with newly prescribed antihypertensive medications. Hypertension 67, 506-512. doi: 10.1161/HYPERTENSIONAHA. 115.06731

KNMP (2006). Royal Dutch Pharmacists Association (KNMP). Nederlandse Apotheeknorm 2006. Available at: https://www.knmp.nl/downloads/nan_2006. pdf [accessed June 5th, 2018]

Kooij, M. J., Heerdink, E. R., van Dijk, L., van Geffen, E. C., Belitser, S. V., and Bouvy, M. L. (2016). Effects of Telephone Counseling Intervention by Pharmacists (TelCIP) on medication adherence; results of a cluster randomized trial. Front. Pharmacol. 7:269. doi: 10.3389/fphar.2016.00269

Kripalani, S., Yao, X., and Haynes, R. B. (2007). Interventions to enhance medication adherence in chronic medical conditions: a systematic review. Arch. Intern. Med. 167, 540-550. doi: 10.1001/archinte.167.6.540
Kronish, I. M., Woodward, M., Sergie, Z., Ogedegbe, G., Falzon, L., and Mann, D. M. (2011). Meta-analysis: impact of drug class on adherence to antihypertensives. Circulation 123, 1611-1621. doi: 10.1161/CIRCULATIONAHA.110.983874

Lam, W. Y., and Fresco, P. (2015). Medication adherence measures: an overview. Biomed. Res. Int. 2015:217047. doi: 10.1155/2015/217047

Lee, J. K., Grace, K. A., and Taylor, A. J. (2006). Effect of a pharmacy care program on medication adherence and persistence, blood pressure, and low-density lipoprotein cholesterol. A randomized controlled trial. JAMA 296, 2563-2571. doi: 10.1001/jama.296.21.joc60162

Leventhal, H., Benyamini, Y., Brownlee, S., Diefenbach, M., Leventhal, E. A., Patrick-Miller, L., et al. (1997). "Illness representations theoretical foundations," in Perceptions of Health and Illness. Current Research and Applications, eds K. J. Petrie and J. A. Weinman (Amsterdam: Harwood Academic Publishers).

Leventhal, H., Diefenbach, M., and Leventhal, E. A. (1992). Illness cognition: using common sense to understand treatment adherence and affect cognition interactions. Cogn. Ther. Res. 16, 143-163. doi: 10.1007/bf01173486

Leventhal, H., Zimmerman, R., and Gutmann, M. (1984). “Compliance: a selfregulation perspective," in Handbook of Behavioral Medicine, ed. D. Gentry (New York, NY: Pergamon Press).

Lin, C. Y., Ou, H. T., Nikoobakht, M., Broström, A., Årestedt, K., and Pakpour, A. H. (2018). Validation of the 5-item medication adherence report scale in older stroke patients in Iran. J. Cardiovasc. Nurs. doi: 10.1097/JCN. 0000000000000488 [Epub ahead of print].

Linn, A. J., Vervloet, M., van Dijk, L., Smit, E. G., and Van Weert, J. C. (2011). Effects of eHealth interventions on medication adherence: a systematic review of the literature. J. Med. Internet Res. 13:e103. doi: 10.2196/jmir.1738

Mardby, A. C., Akerlind, I., and Jorgensen, T. (2007). Beliefs about medicines and self-reported adherence among pharmacy clients. Patient Educ. Couns. 69, 158-164. doi: 10.1016/j.pec.2007.08.011

McDonald, H. P., Garg, A. X., and Haynes, R. B. (2002). Interventions to enhance patient adherence to medication prescriptions: scientific review. JAMA 288, 2868-2879. doi: 10.1001/jama.288.22.2868

Meyer, D., Leventhal, H., and Gutmann, M. (1985). Common-sense models of illness: the example of hypertension. Health Psychol. 4, 115-135. doi: 10.1037/ 0278-6133.4.2.115

Mora, P. A., Berkowitz, A., Contrada, R. J., Wisnivesky, J., Horne, R., Leventhal, H., et al. (2011). Factor structure and longitudinal invariance of the medical adherence report scale-asthma. Psychol. Health 26, 713-727. doi: 10.1080/ 08870446.2010.490585

Morgado, M., Rolo, S., and Castelo-Branco, M. (2011). Pharmacist intervention program to enhance hypertension control: a randomised controlled trial. Int. J. Clin. Pharm. 33, 132-140. doi: 10.1007/s11096-0109474-X

Naderi, S. H., Bestwick, J. P., and Wald, D. S. (2012). Adherence to drugs that prevent cardiovascular disease: meta-analysis on 376,162 patients. Am. J. Med. 125, 882.e1-887.e1. doi: 10.1016/j.amjmed.2011.12.013

Nau, D. P. (2012). Proportion of Days Covered (PDC) as a Preferred Method of Measuring Medication Adherence. Springfield, VA: Pharmacy Quality Alliance.

Nguyen, T. M., La Caze, A., and Cottrell, N. (2016). Validated adherence scales used in a measurement-guided medication management approach to target and tailor a medication adherence intervention: a randomised controlled trial. $B M J$ Open 6:e013375. doi: 10.1136/bmjopen-2016-013375

Nieuwlaat, R., Wilczynski, N., Navarro, T., Hobson, N., Jeffery, R., Keepanasseril, A., et al. (2014). Interventions for enhancing medication adherence (review). Cochrane Database Syst. Rev. 11:CD000011. doi: 10.1002/14651858.CD000011.pub4

Prochaska, J. O., and Velicer, W. F. (1997). The transtheoretical model of health behavior change. Am. J. Health Promot. 12, 38-48. doi: 10.4278/0890-117112.1.38

Rajpura, J. R., and Nayak, R. (2014). Medication adherence in a sample of elderly suffering from hypertension evaluating the influence of illness perceptions, treatment beliefs and illness burden. J. Manag. Care Pharm. 20, 58-65. doi: 10.18553/jmcp.2014.20.1.58

Reynolds, N. R. (2003). The problem of antiretroviral adherence: a selfregulatory model for intervention. AIDS Care 15, 117-124. doi: 10.1080/ 0954012021000039815 
Ross, S., Walker, A., and MacLeod, M. J. (2004). Patient compliance in hypertension: role of illness perceptions and treatment beliefs. J. Hum. Hypertens. 18, 607-613. doi: 10.1038/sj.jhh.1001721

Sabate, E. (2003). World Health Organization. Adherence to Long Term Therapies. Evidence for Action. Geneva: World Health Organization.

Salt, E., Hall, L., Peden, A. R., and Horne, R. (2012). Psychometric properties of three medication adherence scales in patients with rheumatoid arthritis. J. Nurs. Meas. 20, 59-72. doi: 10.1891/1061-3749.20.1.59

SFK (2013). Foundation for Pharmaceutical Statistics (SFK). Royal Dutch Pharmacists Association. Available at: https://www.sfk.nl [accessed June 5th, 2018]

Sjolander, M., Eriksson, M., and Glader, E. L. (2013). The association between patients' beliefs about medicines and adherence to drug treatment after stroke: a cross-sectional questionnaire survey. BMJ Open 3:e003551. doi: 10.1136/ bmjopen-2013-003551

Stewart, K., George, J., Mc Namara, K. P., Jackson, S. L., Peterson, G. M. Bereznicki, L. R., et al. (2014). A multifaceted pharmacist intervention to improve antihypertensive adherence: a cluster-randomized, controlled trial (HAPPy trial). J. Clin. Pharm. Ther. 39, 527-534. doi: 10.1111/jcpt. 12185

Tommelein, E., Mehuys, E., Van Tongelen, I., Brusselle, G., and Boussery, K. (2014). Accuracy of the Medication Adherence Report Scale (MARS-5) as a quantitative measure of adherence to inhalation medication in patients with COPD. Ann. Pharmacother. 48, 589-595. doi: 10.1177/106002801452 2982

Twisk, J. W. R. (2006). Applied Multilevel Analysis: A Practical Guide. Cambridge: Cambridge University Press, 2006. doi: 10.1017/CBO97805116 10806

van de Steeg, N., Sielk, M., Pentzek, M., Bakx, C., and Altiner, A. (2009). Drugadherence questionnaires not valid for patients taking blood-pressure-lowering drugs in a primary health care setting. J. Eval. Clin. Pract. 15, 468-472. doi: 10.1111/j.1365-2753.2008.01038.x

Van der Laan, D. M., Elders, P. J., Boons, C. C., Bosmans, J. E., Nijpels, G., and Hugtenburg, J. G. (2017). The (cost-)effectiveness of a patient-tailored intervention programme to enhance adherence to antihypertensive medication in community pharmacies: study protocol of a randomised controlled trial. Trials 18:29. doi: 10.1186/s13063-016-1696-3

van Dulmen, S., Sluijs, E., van Dijk, L., de Ridder, D., Heerdink, R., and Bensing, J. (2007). Patient adherence to medical treatment: a review of reviews. BMC Health Serv. Res. 7:55. doi: 10.1186/1472-6963-7-55

Vervloet, M., Linn, A. J., van Weert, J. C., de Bakker, D. H., Bouvy, M. L., and van Dijk, L. (2012). The effectiveness of interventions using electronic reminders to improve adherence to chronic medication: a systematic review of the literature. J. Am. Med. Inform. Assoc. 19, 696-704. doi: 10.1136/amiajnl-2011-000748

Vrijens, B., De Geest, S., Hughes, D. A., Przemyslaw, K., Demonceau, J., Ruppar, T., et al. (2012). A new taxonomy for describing and defining adherence to medications. Br. J. Clin. Pharmacol. 73, 691-705. doi: 10.1111/j.1365-2125. 2012.04167.x

Conflict of Interest Statement: The authors declare that the research was conducted in the absence of any commercial or financial relationships that could be construed as a potential conflict of interest.

Copyright (C) 2018 van der Laan, Elders, Boons, Nijpels, van Dijk and Hugtenburg. This is an open-access article distributed under the terms of the Creative Commons Attribution License (CC BY). The use, distribution or reproduction in other forums is permitted, provided the original author(s) and the copyright owner(s) are credited and that the original publication in this journal is cited, in accordance with accepted academic practice. No use, distribution or reproduction is permitted which does not comply with these terms. 